\title{
EMBEDDING DIFFERENTIAL AND SKEW POLYNOMIAL RINGS INTO ARTINIAN RINGS
}

\author{
WILLIAM D. BLAIR AND LANCE W. SMALL \\ (Communicated by Maurice Auslander)
}

\begin{abstract}
Under suitable hypotheses it is shown that a differential or skew polynomial ring can be embedded in an Artinian ring provided that its coefficient ring can be embedded in an Artinian ring.
\end{abstract}

In this paper we continue our recent work on determining when various rings can be embedded in Artinian rings. In [1] it was shown that if $R$ is embeddable in a right Artinian ring and if $\sigma$ is an automorphism of $R$ of finite order, then the skew polynomial ring $R[X ; \sigma]$ is embeddable in a right Artinian ring. We remarked there that we did not know whether the result remains true if we remove the hypothesis that $\sigma$ is of finite order. Here we consider the problem for arbitrary $\sigma$ when $R$ is either a commutative Noetherian ring or a right Noetherian ring which is finitely generated as a module over its center. We also consider the embedding problem when we replace the automorphism by a derivation. We first show that if $R$ is an order in a right Artinian ring and if $\delta$ is a derivation of $R$, then the differential polynomial ring $R[X ; \delta]$ is an order in a right Artinian ring. We use this result to show that if $R$ is embeddable in a right Artinian ring and if (i) $R$ is of characteristic zero and $\delta$ is an arbitrary derivation of $R$ or if (ii) $R$ is of characteristic $p$ and $\delta$ is a nilpotent derivation of $R$, then the differential polynomial ring $R[X ; \delta]$ is embeddable in a right Artinian ring. We also apply our results to note that the generalized Weyl ring $A_{n}(R)$ is embeddable in a right Artinian ring whenever the ring $R$ is embeddable in a right Artinian ring.

\section{DIFFERENTIAL POLYNOMIAL RINGS}

Let $\delta$ be a derivation on the ring $R$. We recall that the differential polynomial ring $R[X ; \delta]$ consists of all polynomials of the form $a_{n} X^{n}+a_{n-1} X^{n-1}+$ $\cdots+a_{1} X+a_{0}$, where $a_{i} \in R$ for $i=0,1, \ldots, n$. Addition is defined as

Received by the editors May 31, 1989 and, in revised form, November 23, 1989.

1980 Mathematics Subject Classification (1985 Revision). Primary 16A05.

This research was supported in part by NSA Grant MDA 904-87-H-2019 (Blair) and NSF Grant DMS-8620068 (Small).

This manuscript is submitted for publication with the understanding that the United States Government is authorized to reproduce and distribute reprints for Governmental purposes. 
usual, and multiplication is given by $X^{i} X^{j}=X^{i+j}$ and $X a=a X+\delta(a)$ for all $a \in R$.

Our first theorem is the analogue for differential polynomial rings of a theorem of [2]. This result is needed for the proof of Theorem 2, but it is also of independent interest. Since our interest here is in the differential polynomial case, we state the theorem for $R[X ; \delta]$, but it is not difficult to modify the proof to handle the case of Ore extensions $R[X ; \sigma, \delta]$, thereby covering both the skew and differential polynomial cases together.

Theorem 1. Let $R$ be an order in a right Artinian ring $S$, and let $\delta$ be a derivation of $R$. Then the differential polynomial ring $R[X ; \delta]$ is an order in a right Artinian ring.

Proof. Using the usual quotient rule, we can extend the derivation $\delta$ from $R$ to a derivation of $S$ which we also call $\delta$. Let $\mathbf{M}$ be the set of all monic polynomials in the right Noetherian ring $S[X ; \delta]$. By Proposition 2.2 of [4], we have that $\mathbf{M}$ is a right denominator set of $S[X ; \delta]$, and we may form the right Noetherian ring $T=S[X ; \delta]_{\mathbf{M}}$. By Theorem 2.4 of [4], the Krull dimension of $T$ is 0 , and so $T$ is a right Artinian ring.

It is easy to check that the set of all regular elements of $R$ form a right denominator set in $R[X ; \delta]$, and so every element of $S[X ; \delta]$ can be shown to have the form $f(X) c^{-1}$ where $f(X) \in R[X ; \delta]$ and $c$ is a regular element of $R$. Furthermore, if $f(X) c^{-1}$ is a regular element of $S[X ; \delta]$, then $f(X)$ is regular in $R[X ; \delta]$. Thus we see that every element of $T$ has the form $f(X) g(X)^{-1}$ where $f(X) \in R[X ; \delta]$ and $g(X)$ is a regular element of $R[X ; \delta]$. Finally, every regular element of $R[X ; \delta]$ is seen to be right regular when considered as an element of the right Artinian ring $T$, and so is invertible in $T$. Thus $R[X ; \delta]$ is a right order in $T$.

Theorem 2. Let $R$ be a ring with no $\mathbb{Z}$-torsion, and let $\delta$ be a derivation of $R$. If $R$ is embeddable in a right Artinian ring, then $R[X ; \delta]$ is embeddable in a right Artinian ring.

Proof. Let $R$ be embeddable in the right Artinian ring $S$. If $S$ has any $\mathbb{Z}$ torsion, we may factor out the ideal of all $\mathbb{Z}$-torsion elements from $S$, and $R$ will embed in the factor ring. Hence without loss of generality we may assume that $S$ has no $\mathbb{Z}$-torsion, and since $S$ is right Artinian we may assume $S$ is a $\mathbb{Q}$-algebra with $\delta(1)=0$.

Form the ring $T=S[[Y]]$ of formal power series over $S$. The ordinary derivative $D=\frac{d}{d Y}$, given by $D\left(\sum_{j=0}^{\infty} s_{j} Y^{j}\right)=\sum_{j=1}^{\infty} j s_{j} Y^{j-1}$, is a derivation on $T$. By Theorem 2 of [6], $T$ is a right order in a right Artinian ring $Q=Q(T)$.

Following [3], we embed $R$ into $T$ via the Taylor map $\tau: R \rightarrow T$ given by

$$
\tau(r)=\sum_{j=0}^{\infty} \frac{1}{j !} \delta^{j}(r) Y^{j}
$$


Clearly $\tau$ is additive and since

$$
\delta^{j}\left(r_{1} r_{2}\right)=\sum_{i=0}^{j}\left(\begin{array}{l}
j \\
i
\end{array}\right) \delta^{i}\left(r_{1}\right) \delta^{j-i}\left(r_{2}\right),
$$

the Leibniz rule, we have $\tau\left(r_{1} r_{2}\right)=\tau\left(r_{1}\right) \tau\left(r_{2}\right)$. Clearly $\tau$ is one-to-one. Since $\delta(1)=0$, we have $\tau(1)=1$. Hence $\tau$ is a ring monomorphism. Next observe that $D \tau=\tau \delta$, and so $\tau$ induces a ring monomorphism $\tau^{*}: R[X ; \delta] \rightarrow$ $T[Z ; D]$ given by $\tau^{*}\left(\sum_{i=0}^{n} r_{i} X^{i}\right)=\sum_{i=0}^{n} \tau\left(r_{i}\right) Z^{i}$.

Since $T$ is a right order in a right Artinian ring, Theorem 1 implies that $T[Z ; D]$ is a right order in a right Artinian ring, and so $R[X ; \delta]$ embeds in this right Artinian ring.

We next make an easy but useful observation.

Observation 3. Let $T \supseteq R$ be rings. If $T_{R}$ is a finitely generated free right $R$-module and if $R$ embeds in a right Artinian ring, then $T$ embeds in a right Artinian ring.

Theorem 4. Let $R$ be a ring of characteristic $p$, and let $\delta$ be a nilpotent derivation on $R$. If $R$ embeds in a right Artinian ring, then $R[X ; \delta]$ embeds in a right Artinian ring.

Proof. Suppose $\delta^{m}=0$. Let $p^{n} \geq m$. Then $\delta^{p^{n}}=0$. Since for all $k \in \mathbb{Z}^{+}$, $X^{k} a=\sum_{j=0}^{k}\left(\begin{array}{l}k \\ j\end{array}\right) \delta^{j}(a) X^{k-j}$ we have $X^{p^{n}} a=a X^{p^{n}}+\delta^{p^{n}}(a)=a X^{p^{n}}$. Thus the subring $S$ of $R[X ; \delta]$ consisting of all polynomials of the form $a_{t} X^{p^{n} t}+$ $a_{t-1} X^{p^{n}(t-1)}+\cdots+a_{1} X^{p^{n}}+a_{0}$ is isomorphic to an ordinary polynomial ring over $R$ and as such is embeddable in a right Artinian ring. Since $R[X ; \delta]$ is a finitely generated free right module over $S$, we have that $R[X ; \delta]$ embeds in a right Artinian ring by Observation 3.

We remark that it is not known whether Theorem 4 remains true if we delete the hypothesis that $\delta$ is nilpotent.

Let $R$ be a ring; the first Weyl ring over $R$ is defined by

$$
A_{1}(R)=R\left[Y_{1}\right]\left[X_{1} ; \frac{\partial}{\partial Y_{1}}\right]
$$

and the $n$th Weyl ring over $R$ is

$$
A_{n}(R)=A_{n-1}(R)\left[Y_{n}\right]\left[X_{n} ; \frac{\partial}{\partial Y_{n}}\right] .
$$

As a further application of Theorem 1, we note that if the ring $R$ is a right order in a right Artinian ring, then $A_{n}(R)$ is a right order in a right Artinian ring; or, if the ring $R$ is embeddable in a right Artinian ring, then $A_{n}(R)$ is also embeddable in a right Artinian ring.

\section{SKEW POLYNOMIAL RINGS}

Let $\sigma$ be an automorphism of the ring $R$. We recall that the skew polynomial ring $R[X ; \sigma]$ consists of all polynomials of the form $a_{n} X^{n}+a_{n-1} X^{n-1}+\cdots+$ $a_{1} X+a_{\mathrm{c}}$, where $a_{i} \in R$ for $i=0,1, \ldots, n$. Addition is defined as usual, and 
multiplication is given by $X^{i} X^{j}=X^{i+j}$ and $X a=\sigma(a) X$ for all $a \in R$.

Although our next result follows immediately from Theorem 8 , we prefer to prove it first since the idea of the proof is more transparent in the special case.

Theorem 5. Let $R$ be a commutative Noetherian ring and let $\sigma$ be an automorphism of $R$. Then the skew polynomial ring $R[X ; \sigma]$ is embeddable in a right Artinian ring.

Proof. By Noetherian induction, we may assume that all proper factor rings $\bar{R}$ of $R$ have the property that for any automorphism $\alpha$ of $\bar{R}$ we have that $\bar{R}[X ; \alpha]$ is embeddable in a right Artinian ring.

Let $Q_{1} \cap Q_{2} \cap \cdots \cap Q_{t}=0$ be a reduced primary decomposition of 0 , and let $P_{j}$ be the prime ideal associated to $Q_{j}$ for $j=1, \ldots, t$. Now for each $j$ there exists $m_{j} \in \mathbb{Z}^{+}$such that $P_{j}^{m_{j}} \subseteq Q_{j}$. Since $\sigma$ is an automorphism of $R$ and since the associated prime ideals of 0 are uniquely determined, we know that $\sigma$ acts as a permutation on the set $\left\{P_{1}, P_{2}, \ldots, P_{t}\right\}$, and so $\sigma$ has finite order as a permutation on this set. Thus there exists $n \in \mathbb{Z}^{+}$so that $\sigma^{n}\left(P_{j}\right)=P_{j}$ for $j=1, \ldots, t$. Clearly $\sigma^{n}\left(P_{j}^{m_{j}}\right)=P_{j}^{m_{j}}$ for all $j$.

If $P_{j}^{m_{j}} \neq 0$ for all $j$, then $R / P_{j}^{m_{j}}$ is a proper factor ring of $R$ for each $j$, and $\sigma^{n}$ induces an automorphism on $R / P_{j}^{m_{j}}$ for each $j$. Since $R \hookrightarrow$ $R / P_{1}^{m_{1}} \oplus \cdots \oplus R / P_{t}^{m_{t}}$, the skew polynomial ring $R\left[Y ; \sigma^{n}\right]$ embeds in the direct sum of the skew polynomial rings $R / P_{j}^{m_{j}}\left[Y ; \sigma^{n}\right]$, and each of these embeds in a right Artinian ring by assumption. Now the subring $T$ of $R[X ; \sigma]$ consisting of all polynomials of the form $a_{t} X^{n t}+a_{t-1} X^{n(t-1)}+\cdots+a_{1} X^{n}+a_{0}$ is isomorphic to $R\left[Y ; \sigma^{n}\right]$, and so $T$ embeds in a right Artinian ring. By Observation 3 , $R[X ; \sigma]$ embeds in a right Artinian ring since it is a finitely generated free module over $T$.

Now suppose $P_{j}^{m_{j}}=0$ for some $j$, say $j=1$. Since $P_{1}$ is a nilpotent prime ideal, it is the prime radical of $R$, and $P_{1}$ is the only minimal prime ideal of $R$. Since the primary ideals associated to 0 whose corresponding prime ideals are isolated are uniquely determined and since $Q_{1}$ is the only such primary ideal, we have $\sigma\left(Q_{1}\right)=Q_{1}$ and so $\sigma^{n}\left(Q_{1}\right)=Q_{1}$. Also $\sigma^{n}\left(P_{j}^{m_{j}}\right)=P_{j}^{m_{j}}$ for $j=2, \ldots, t$. Thus if $Q_{1} \neq 0$ then $\sigma^{n}$ induces an automorphism on the proper factor ring $R / Q_{1}$. We may now repeat the argument of the previous paragraph.

If, on the other hand, $Q_{1}=0$, then $R$ has prime radical $N(R)=P_{1}$, and the regularity condition of [5] holds. Thus $R$ is an order in an Artinian ring, and then by [2] we have that $R[X ; \sigma]$ is an order in a right Artinian ring.

We recall that an ideal is called irreducible if we cannot write $I=J \cap K$ where $J$ and $K$ are ideals which properly contain $I$. 
Lemma 6. Let $A$ be a right Noetherian ring with center $R$, and let $I$ be an irreducible ideal of $A$. Let $b \in R$ such that $b^{j} \notin I$ for all $j \in \mathbb{Z}^{+}$, and let $a \in A$. If $a b \in I$, then $a \in I$.

Proof. The standard commutative proof works.

Corollary 7. Let $A$ be a right Noetherian ring with center $R$. If $I$ is an irreducible ideal of $A$, then $I \cap R$ is a primary ideal of $R$.

Theorem 8. Let $A$ be a right Noetherian ring which is a finitely generated module over its center $R$, and let $\sigma$ be an automorphism of $A$. Then the skew polynomial ring $A[X ; \sigma]$ embeds in a right Artinian ring.

Proof. By Noetherian induction, we may assume that for any automorphism $\alpha$ of any proper factor ring $\bar{A}$ of $A$ we have that $\bar{A}[Y ; \alpha]$ embeds in a right Artinian ring.

Write $I_{1} \cap \cdots \cap I_{t}=0$, where $I_{j}$ is an irreducible ideal of $A$ for $j=$ $1, \ldots, t$. By Corollary 7, each $I_{j} \cap R$ is a primary ideal of $R$. We may refine $0=\left(I_{1} \cap R\right) \cap \cdots \cap\left(I_{t} \cap R\right)$ to a reduced primary decomposition of 0 in $R$; say $0=Q_{1} \cap \cdots \cap Q_{k}$ is such a refinement, where $Q_{j}$ is a primary ideal with associated prime ideal $P_{j}$. Recall that the reduced decomposition is obtained from $0=\left(I_{1} \cap R\right) \cap \cdots \cap\left(I_{t} \cap R\right)$ by collecting all the $I_{j} \cap R$ which have the same associated prime and discarding any $I_{j} \cap R$ which contains an intersection of other $I_{j} \cap R$. Thus each $Q_{i}$ is contained in some $I_{j} \cap R$, and each $I_{j} \cap R$ contains an intersection of some of the $Q_{i}$ 's. For each $j=1, \ldots, k$ there exists $m_{j} \in \mathbb{Z}^{+}$such that $P_{j}^{m_{j}} \subseteq Q_{j}$, and since $\sigma(R)=R,\left.\sigma\right|_{R}$ is an automorphism of $R$, and thus, as in the proof of Theorem 5, there exists $n \in \mathbb{Z}^{+}$ such that $\sigma^{n}\left(P_{j}^{m_{j}}\right)=P_{j}^{m_{j}}$ for all $j$. Hence $\sigma^{n}\left(P_{j}^{m_{j}} A\right)=P_{j}^{m_{j}} A$, an ideal of $A$. Note that $\bigcap_{j=0}^{k} P_{j}^{m_{j}} A \subseteq \bigcap_{j=0}^{t} I_{j}=0$. If each $P_{j}^{m_{j}} \neq 0$, we proceed as in the proof of Theorem 5 .

If some $P_{j}^{m_{j}}=0$, say $j=1$, then $P_{1}$ is the prime radical of $R$. Let $I$ be any $I_{j}$ which gives rise to $P_{1}$. If $c \in R, c \notin P_{1}$ then $c^{j} \notin I$ for all $j \in \mathbb{Z}^{+}$. Thus $T=\left\{a \in A \mid a c=0\right.$ for some $\left.c \in R, c \notin P_{1}\right\} \subseteq I$ by Lemma 8. Since $\sigma\left(P_{1}\right)=P_{1}$, we have $\sigma(T)=T$ and so $\sigma^{n}(T)=T$, and we can use $T$ instead of $P_{1}^{m_{1}} A$ in the argument, provided $T \neq 0$. If, on the other hand, $T=0$, then $R$ satisfies the regularity condition of [5], and $A$ is torsion-free over $R$. By Theorem 3.11 of [5] we have that $A$ is an order in a right Artinian ring, and we may apply [2] as in the proof of Theorem 5.

\section{REFERENCES}

1. W. D. Blair and L. W. Small, Embeddings in Artinian rings and Sylvester rank functions, Israel J. Math. 58 (1987), 10-18.

2. A. V. Jategaonkar, Skew polynomial rings over orders in Artinian rings, J. Algebra 21 (1972), 51-59.

3. D. Quinn, Embeddings of differential operator rings and Goldie dimension, Proc. Amer. Math. Soc. 102 (1988), 9-16. 
4. R. Resco, L. W. Small, and J. T. Stafford, Krull and global dimensions of semiprime Noetherian PI-rings, Trans. Amer. Math. Soc. 274 (1982), 285-295.

5. L. W. Small, Orders in Artinian rings, J. Algebra 4 (1966), 13-41.

6. __ Orders in Artinian rings II, J. Algebra 9 (1968), 266-273.

Department of Mathematical Sciences, Northern Illinois University, DeKalb, IlliNOIS 60115

Department of Mathematics, University of California, San Diego, la Jolla, CaliforNIA 92093 